\title{
Aging and Speech Understanding
}

\author{
Ji Young Lee \\ Department of Audiology and Speech-Language Pathology, Catholic University of Daegu, Gyeongsan, Korea
}

Received March 26, 2015

Revised April 4,2015

Accepted April 5, 2015

\begin{abstract}
As people age, structural as well as neural degeneration occurs throughout the auditory system. Many older adults experience difficulty in understanding speech especially in adverse listening conditions although they can hear speech sounds. According to a report of the Committee on Hearing and Bioacoustics and Biomechanics of the National Research Council, peripheral, central-auditory, and cognitive systems have long been considered major factors affecting the understanding of speech. The present study aims to review 1) age-related changes in the peripheral, central-auditory, and cognitive systems, 2) the resulting decline in the understanding of speech, and 3) the clinical implication for audiologic rehabilitation of older adults. Once the factors affecting the understanding of speech in older adults are identified and the characteristics of age-related speech understanding difficulties are examined, clinical management could be developed for prevention and treatment. Future research about problems related to the understanding of speech in older adults will help to improve the quality of life in the elderly.

J Audiol Otol 2015;19(1):7-13

KEY WORDS: Aging · Peripheral auditory system · Central auditory system · Cognition Speech understanding.
\end{abstract}

\section{Introduction}

Aging causes a variety of biochemical and molecular changes including DNA damage, mitochondrial decay, reduction of cellular water concentration, metabolic and vascular dysfunction, and decrease in cellular membrane elasticity [1]. As a result, such changes have a detrimental effect on the auditory function of aging individuals. As people age, structural and neural degeneration occurs throughout the auditory system due to a loss of sensory cells and cochlear neurons, reduction in the number of synaptic contacts, changes in the conduction of the cochlear duct, changes in excitatory and inhibitory neurotransmitter systems, etc [2-4].

Many older adults experience difficulty understanding speech, especially in demanding listening conditions, although they can hear speech sounds. There are large individual differences, however, in speech recognition performance among older adults. According to a report from the Committee on

This is an Open Access article distributed under the terms of the Creative Commons Attribution Non-Commercial License (http://creativecommons. org/licenses/by-nc/3.0/) which permits unrestricted non-commercial use, distribution, and reproduction in any medium, provided the original work is properly cited.
Hearing and Bioacoustics and Biomechanics of the National Research Council [5], peripheral, central-auditory, and cognitive systems have long been considered major factors affecting speech understanding [6,7]. The functions of the peripheral and central-auditory systems are reflected in hearing sensitivity measured by the conventional pure-tone audiometer and speech audiometric measures, respectively. The cognitive function includes various mental processing such as attention, memory, perception, and executive function, which are closely related to speech understanding [8].

As adults are now surviving longer, more attention is focused on the hearing and speech problems of older adults. Therefore, better understanding of the underlying mechanisms, characteristics, and clinical implications of speech understanding in older adults is needed. The present study aims to review 1) age-related changes in the peripheral, central-auditory, and cognitive systems, 2) the resulting decline of speech understanding in terms of decreased hearing sensitivity, psychoacoustic performance, and speech understanding performance, and 3) clinical implications for audiologic rehabilitation of older adults. 


\section{Age-Related Changes in Peripheral, Central-Auditory, and Cognitive Systems}

\section{Peripheral auditory system}

The peripheral auditory system includes the outer ear through the inner ear and the auditory nerve. Although age-related changes in the outer and middle ear may occur in the form of collapse of the cartilaginous external auditory canal or stiffening of the tympanic membrane and ossicular chain, their effects do not noticeably cause hearing problems [3]. Age-related changes in the cochlear, on the other hand, significantly affect the transduction of sound into neural impulses, resulting in loss of some of the information from speech sounds. The sensory cells in the cochlea are generated within the first trimester of development and must survive throughout the individual's lifetime. Because a small number of hair cells correspond to each frequency region within the cochlear and regeneration does not occur, the loss of hair cells have dramatic effects on hearing [9]. While high frequency loss commonly results from the loss of the inner hair cells at the basal turn of the cochlea, the loss of outer hair cells may reduce the active feedback loop activity, possibly causing hearing loss, limited frequency selectivity, and a reduced dynamic range. Also, aging can cause a decrease in the volume of strial tissue on the lateral cochlear wall, resulting in hearing loss $[4,10]$. The stria vascularis produces endolymph, maintaining the endocochlear potetial of $+80 \mathrm{mV}$ for the scala media. Animal models indicate that age-related deterioration of the strial tissue on the lateral cochlear wall correlates with a reduction in the endocochlear potential $[10,11]$.

As we age, hearing sensitivity declines gradually and progressively. Presbycusis refers to the loss of hearing that occurs with aging. It usually occurs in both ears. The reduced hearing sensitivity begins with high frequency sounds and advances to low frequency sounds. At an early stage, it does not noticeably interfere with speech understanding. With advancing age, however, speech understanding is affected by the diminished hearing sensitivity. While young children can hear frequencies as high as $20 \mathrm{kHz}$, the hearing threshold of most adults decreases rapidly above $15 \mathrm{kHz}$ [12].

\section{Central auditory system}

The central auditory system consists of various nuclei that relay information from the cochlea and auditory nerve to other nuclei in the auditory system. The transfer of neural impulses from the peripheral to the central auditory system occurs at the cochlear nuclei. As afferent pathways pass from the cochlea to the higher auditory centers, the neural signal is con- verted to sound and later analyzed by the cognitive centers of the brain. Efferent pathways play a role in regulating function at lower levels. Because bilateral processing of auditory information occurs at all levels above the cochlea, hearing is rarely impaired after stroke even though the understanding of sound can be impaired [9]. The superior olivary complex is involved with binaural processing and the acoustic reflex, and the lateral lemniscus contains both ascending and descending pathways. The inferior colliculus, the largest auditory structure, is considered to be involved with sound localization and frequency resolution. After the auditory information is sent to the temporal lobe in two pathways at the thalamus level, primary auditory reception occurs in Heschl's gyri in either hemisphere. While auditory linguistic processing occurs primarily in the left hemisphere at Wernicke's and the insular cortex, nonlinguistic information is processed primarily in the right hemisphere. Pathways from both hemispheres interact with each other by stimulating, inhibiting, and transferring information $[13,14]$.

With aging, the number of neurons and the size of cells in each auditory nucleus decrease, and the neuronal population in the auditory cortex is dramatically reduced [15]. These changes in the central auditory system often result from hearing loss or attenuation of neural input from the peripheral auditory system. A number of pathologies accompanying aging such as arteriosclerosis, anoxia, and metabolic dysfunction of the liver or renal system may also affect the central auditory pathways. Usually, simple amplification with a hearing aid does not efficiently improve the decreased processing of complex acoustic stimuli in the central auditory system. Moreover, a loss of neural synchrony or disruption of binaural processing in the central auditory system may lead to the loss of further auditory information and degrade the input to the cognitive level engaged in lexical access [16].

The age-related change in central auditory processing, which results in poor speech understanding, is referred to as central presbycusis. In the traditional view, central presbycusis is attributed to alterations of the auditory portions of the central nervous system. In the broader view, however, the term is also used to include not only central auditory but also amodal cognitive degradations affecting auditory processing and speech perception [8].

\section{Cognitive system}

Although the higher centers in the central auditory system are closely associated with cognition, cognition is differentiated from the central auditory system. Cognition affects not only the auditory modality but also other sensory modalities. As time progresses, cognitive performance is also reduced. 
Various biological systems must be in harmony to maintain optimal cognitive function. Aging may cause chronic inflammation, oxidative stress, insulin resistance, decrease in hormone levels, and endothelial dysfunction, which can perturb the biological system and deteriorate the brain and subsequent cognitive function [17]. The resulting biological deterioration includes reduction in brain volume, loss of myeline integrity, cortical thinning, and impaired serotonin and acetylcholine [18,19].

Aging is associated with the decrease in cognitive functions such as attention, memory, perception, language processing, decision making, and executive function. Of these, attention and working memory are the most basic cognitive functions affecting speech understanding in older adults. Attention is usually involved in all other cognitive functions unless the response to a task has become habitual or automatic. There are some subdivisions of attention, such as selective attention which enables us to focus on a specific task while ignoring other irrelevant tasks, divided attention which permits us to attend to two or more tasks simultaneously, and sustained attention which allows us to maintain concentration on a task over time. Older adults showed the increased engagement of frontal lobe systems in difficult listening situations, reflecting greater attentional burden. These findings indicate the effect of attention deficits on speech understanding. Working memory is also considered crucial for speech processing. Conceptually, working memory is not quite distinct from short-term memory. Where short-term memory refers to a cognitive capacity for retaining information such as digits, words, and names, working memory is defined as a cognitive capacity involving the active manipulation of information before recall. Adults with better working memory showed better speech perception in noise than those with poorer working memory [19], suggesting that age-related deficits in working memory may have a harmful effect on speech understanding in older adults.

Interaction of peripheral, central-auditory, and cognitive systems

The peripheral, central-auditory, and cognitive systems may work in isolation or together. The impact of each factor is highly variable. Because peripheral hearing loss often accompanies advanced age, it is difficult to rule out the influence of hearing loss on central auditory processing even in well-controlled studies [20]. There is little evidence to support declines in central auditory function independent of hearing loss or cognitive function [8,21]. For instance, age-related changes in the central auditory system may result from the deficit in the peripheral auditory system. Also, cognitive deficits can exacerbate declines in hearing sensitivity, further contributing to the older adult's speech recognition problem in noise [22]. Age-related changes in the central auditory system may further degrade the input to the cognitive level [16]. Though a specific test is administered to measure the peripheral or central-auditory processing system, the test results cannot be separated from the cognitive function.

\section{Age-Related Decline of Speech Understanding}

\section{Hearing sensitivity}

Hearing sensitivity is one of the main causes for speech understanding deterioration in older adults though speech understanding is not directly predicted by their pure-tone audiograms [23]. It is apparent that older adults tend to experience more difficulties in understanding speech than is predicted by hearing sensitivity even when their hearing thresholds match those of younger adults. To predict future hearing loss, it may be useful to measure hearing sensitivity in the extended high frequencies (EHF) up to $16-20 \mathrm{kHz}$ because older adults may have greater decreases at EHFs above $8000 \mathrm{~Hz}$ than at the lower audiometric frequencies of $250-8000 \mathrm{~Hz}$ [24]. Males have poorer thresholds than females at $9-14 \mathrm{kHz}$ but the thresholds of males are no different from those of females at $16-20 \mathrm{kHz}$ [25]. EHF thresholds are highly correlated with thresholds at $4 \mathrm{kHz}$ and $8 \mathrm{kHz}$. Early monitoring of hearing thresholds at EHF may be useful to predict the onset of hearing loss and recommend strategies for prevention.

\section{Psychoacoustic performance}

The reduced speech understanding of older adults may be attributed to poor psychoacoustic performance. Many psychoacoustic measures have demonstrated poorer performance by older adults in temporal, frequency, or intensity processing compared to younger adults. Because accurate temporal and spectral processing is crucial for identifying the signal in noise, deficits in spectrotemporal processing may cause older adults to experience difficulties with speech understanding in real life.

In particular, temporal processing in older adults has been widely studied in a variety of tasks such as gap detection, duration discrimination, intensity discrimination, and forward or backward masking. It is conceivable that the age-related deficit in following fast speech may be associated with the slow processing of acoustic information. Temporal processing research uses tonal signals instead of speech signals to minimize the effects of speech or language processing and control psychoacoustic parameters more precisely [26]. Gap detection research has demonstrated that older adults need longer gaps than young adults [27]. When gap detection was examined, 
matching young and older adults with normal hearing sensitivity, older adults exhibited larger gap detection thresholds than young adults [28]. In a study of duration discrimination, older adults showed slightly larger just noticeable differences for stimulus duration compared to young adults with matched hearing sensitivity. Age effects on duration discrimination increases as stimulus complexity increases [29]. For intensity discrimination, older adults with normal hearing had poorer performance at lower frequencies than young adults. In a spectrotemporal model of auditory processing, intensity discrimination at lower frequencies is based on temporal cues, whereas intensity discrimination at higher frequencies is dominated by spectral cues. Thus, poor intensity discrimination at lower frequencies for older adults may indicate a deficit in temporal processing [30]. In a backward masking temporal processing task, young adults with normal hearing showed less overall masking and steeper recovery functions compared to older adults with normal hearing. Older adults showed significant masking even at the longest delay of $20 \mathrm{~ms}$ [31].

\section{Speech understanding performance}

Many older adults experience difficulty in understanding speech when the speech is accompanied by noise, when the speech rate is fast, or when the amount of information is loaded even if they do not have hearing loss [26]. These difficulties cannot be explained simply by audiometric test results because older adults have disadvantages not only in peripheral but also in central-auditory and cognitive functions [32].

The rate of speech is a factor that affects speech understanding in older adults. Older adults usually show poorer perception of rapid or time-compressed speech compared to young adults. The word recognition score of older adults decreases as speech rate increases whereas word recognition in young adults is hardly affected by speech rate, reflecting a slow perceptual process with aging. Time compression is one of the most widely used methods for altering the rate of speech while preserving the other properties of the original speech stimuli. The resulting temporal aspects are determined by how much the speech signal is reduced (compression rate), how often portions of the speech signal are discarded (compression frequency), and how long the discarded interval is (discarded interval). Time-compression of speech at a rate of 60 percent eliminates 60 percent of the speech. The remaining 40 percent may be very different, depending on the length of the discard intervals [33]. When subjects reported associations among the items addressed in time-compression passages manipulated at three compression ratios of 30,45 , and $60 \%$, and at seven discard interval lengths from 35 to $155 \mathrm{msec}$, older adults were more affected by compres- sion ratio and discard interval length than young adults. The age-related deficit in time-compressed speech is greater in noisy than in quiet environments [34].

Usually, older adults do not have problems with understanding speech in quiet environments or with familiar speakers. They are particularly vulnerable to the effects of noise on speech understanding. They experience difficulty following conversations in adverse listening situations including noise and reverberation. Successful speech understanding in noisy situations is important for social, vocational, and educational activities in everyday life. Older adults experience more difficulty with understanding speech in noisy environments than younger adults due to decreased hearing sensitivity and reduced attention [32]. Understanding speech in noisy situations is a complex task involving the interplay of sensory, central-auditory, and cognitive processes. The relationship between pure-tone threshold and speech understanding in noisy environments is weak in older adults. The audiogram cannot accurately explain speech understanding performance, particularly when speech is accompanied by noise [35]. Numerous studies have been conducted to examine what factors affect age-related deficits in speech understanding in noisy environments, with a number of variables such as the audibility of the speech signal, the type of speech signal, the type of noise, and the signal-to-noise ratio. Regarding the audibility of the speech signal, speech understanding in noisy environments was lower even in older adults with normal hearing thresholds [36]. Data from 3189 adults ranging in age from 48 to 92 years demonstrated that monosyllabic word recognition scores in older adults using competing messages were poorer compared to younger adults. The data also indicated that word recognition performance was poorer for males than women in each age decade, even after statistically adjusting for hearing sensitivity [25]. Regarding the type of noise, older adults had more difficulty with understanding speech in babble noise than in a steady-state noise compared to younger adults [37]. Given that cognitive processing declines with aging, babble noise including cognitively meaningful information may degrade speech understanding more severely for older than for younger adults.

\section{Clinical Implications for Audiologic Rehabilitation of Older Adults}

\section{Use of devices}

A hearing aid can be an important starting point for the audiologic rehabilitation of older adults with hearing impairment. Hearing aids significantly improve speech recognition for older adults both in quiet and noisy environments but they do not 
reduce the perceived effort [38]. Older hearing aid users with mild to moderate hearing loss exhibited a positive effect of hearing aids on self-perceived hearing but did not show the effect of the hearing aids on social activities, satisfaction with social relations, well-being, and cognitive functioning. Despite the benefits of amplification for older adults, many refuse to wear hearing aids for reasons such as cost, poor benefit, stigma, low self-esteem, and denial of hearing loss. Satisfaction in hearing aid users was higher for more technologically advanced devices [39].

Cochlear implants are also beneficial for older adults with severe to profound hearing loss. In quiet environments, older adults had better speech recognition scores after cochlear implantation than preimplantation [40]. People implanted at 70 or older demonstrated significantly improved speech recognition performance compared to speech recognition performance prior to implantation. Their improvement was, however, less than younger individuals with cochlear implants when their performance was measured at the same time period after implantation [41]. The speech therapist's opinion, the patient's satisfaction, and the hours of use for the cochlear implants were similar in the older and young adults evaluated, indicating that age is not a contraindication for cochlear implantation [42].

Technological advances have led to the development of assistive listening devices that can provide additional benefits to hearing aids. Assistive listening devices can improve the signal-to-noise ratio in adverse listening situations. FM receivers built into behind-the-ear hearing aids or personal FM systems are now available, making FM systems more portable and easier to use [26]. When twelve older adults fitted with behind-the-ear hearing aids with built-in FM receivers were instructed to repeat test words, phoneme recognition scores in noisy environments with an FM system equaled phoneme recognition scores in quiet environments with a hearing aid [43]. Also, closed captioning can be used to help older adults with hearing impairment access television or theater movies more comfortably, although it was originally developed for deaf people. Future research is needed to verify its benefits for older adults with hearing impairment [26].

\section{Effects of training}

Although hearing aids or assistive listening devices increase audibility, they cannot compensate for deficits in auditory processing and speech perception. Short-term or long-term training have provided a strategy to ameliorate these deficits in older adults. The training program usually consists of multimodal tasks, integrating auditory and visual attention, working memory, etc., because speech understanding in older adults is strongly affected by central-auditory and cognitive processing as well as hearing sensitivity. Short-term auditory training has led to improvements in neural response timing and frequency representation in both children and young adults. Older adults demonstrated improved auditory memory and speed in auditory processing after short-term auditory training. Also, there is evidence that long-term auditory training can improve neural timing in older adults [44].

There is evidence of induced neural plasticity after training in older adults. In a study of short-term auditory based cognitive training, older adults with bilateral pure-tone averages below $40 \mathrm{~dB}$ HL received in-home training for 40 hours over 8 weeks. The training program consisted of six adaptive exercises including discriminating between frequency-modulated sweeps, discriminating between syllables, repeating syllables and words, matching pairs of syllables and words, remembering commands, and remembering stories. After training, older adults showed a partial reversal of age-related neural timing delays, exhibiting improved neural timing, memory, speed of processing, and speech perception in noisy situations [44]. To examine the efficacy of a novel computerized cognitive training program in older adults, older adults aged 65 and older were randomized to the experimental group to receive a novel brain plasticity-based computerized cognitive training program or the control group to receive a general cognitive stimulation program. After 8 weeks, the experimental group showed greater auditory memory and attention compared to the control group. The results showed that the effect of the novel cognitive training program was generalized to untrained measures of auditory memory and attention, suggesting that the improved performance after training also affected systems serving auditorybased cognition [45].

\section{Conclusions}

The lifespan of people is increasing with improved nutrition and healthcare. Consequently, more attention is focused on the quality of life for older adults. Many older adults have difficulties with understanding speech, especially in difficult listening conditions. The decrease in speech understanding affects their everyday life as they may avoid social, vocational, and educational activities because of communication difficulties. Recent investigations have sought to elucidate the factors that affect speech understanding. In the present study, the peripheral, central-auditory, and cognitive systems were considered important factors affecting speech understanding in older adults. Age-related changes in these three factors cause deficits in hearing sensitivity, psychoacoustic performance, and speech understanding performance. In addition to devic- 
es such as hearing aids, cochlear implants, and assistive listening devices, audiologic rehabilitation would need to include integrative and multimodal training programs. Once the factors affecting speech understanding in older adults are identified and the characteristics of age-related speech understanding difficulties are examined, clinical management procedures could be developed for prevention and treatment. A multidisciplinary approach is needed to better understand the underlying mechanisms, characteristics, and clinical implications of speech understanding in older adults.

\section{Acknowledgments}

This study was supported by research grants from the Catholic University of Daegu.

\section{REFERENCES}

1) Seidman MD, Ahmad N, Joshi D, Seidman J, Thawani S, Quirk WS. Age-related hearing loss and its association with reactive oxygen species and mitochondrial DNA damage. Acta Otolaryngol Suppl 2004: 16-24.

2) Belal A Jr, Glorig A. The ageing ear. A clinico-pathological classification. J Laryngol Otol 1987;101:1131-5.

3) Chisolm TH, Willott JF, Lister JJ. The aging auditory system: anatomic and physiologic changes and implications for rehabilitation. Int $\mathrm{J}$ Audiol 2003;42 Suppl 2:2S3-10.

4) Schuknecht HF, Gacek MR. Cochlear pathology in presbycusis. Ann Otol Rhinol Laryngol 1993;102(1 Pt 2):1-16.

5) Speech understanding and aging. Working Group on Speech Understanding and Aging. Committee on Hearing, Bioacoustics, and Biomechanics, Commission on Behavioral and Social Sciences and Education, National Research Council. J Acoust Soc Am 1988;83:859-95.

6) Gordon-Salant S, Fitzgibbons PJ, Yeni-Komshian GH. Auditory temporal processing and aging: implications for speech understanding of older people. Audiol Res 2011;1:9-15.

7) Humes LE. Speech understanding in the elderly. J Am Acad Audiol 1996;7:161-7.

8) Humes LE, Dubno JR, Gordon-Salant S, Lister JJ, Cacace AT, Cruickshanks KJ, et al. Central presbycusis: a review and evaluation of the evidence. J Am Acad Audiol 2012;23:635-66.

9) Howarth A, Shone GR. Ageing and the auditory system. Postgrad Med J 2006;82:166-71.

10) Schulte BA, Schmiedt RA. Lateral wall Na, K-ATPase and endocochlear potentials decline with age in quiet-reared gerbils. Hear Res 1992;61:35-46.

11) Gratton MA, Schmiedt RA, Schulte BA. Age-related decreases in endocochlear potential are associated with vascular abnormalities in the stria vascularis. Hear Res 1996;94:116-24.

12) Ashihara K, Kurakata K, Mizunami T, Matsushita K. Hearing threshold for pure tones above $20 \mathrm{kHz}$. Acoust Sci Technol 2006;27:12-9.

13) Becker JT, Boller F, Lopez OL, Saxton J, McGonigle KL. The natural history of Alzheimer's disease. Description of study cohort and accuracy of diagnosis. Arch Neurol 1994;51:585-94.

14) Musiek FE, Weihing JA, Oxholm VB. Anatomy and physiology of the central auditory nervous system: a clinical perspective. In: Roeser RJ, Valente M, Hosford-Dunn H, editors. Audiology: Diagnosis. 2nd ed. NY: Thieme;2011. p.37-64.

15) Willott JF. Aging and the auditory system. San Diego, CA: Singular Publishing Group;1991.

16) Rönnberg J, Rudner M, Foo C, Lunner T. Cognition counts: a working memory system for ease of language understanding (ELU). Int J Audiol 2008;47 Suppl 2:S99-105.
17) Aruoma OI, Neergheen VS, Bahorun T, Jen LS. Free radicals, antioxidants and diabetes, embryopathy, retinopathy, neuropathy, nephropathy and cardiovascular complications. Neuroembryol Aging 2007; 4:117-37.

18) Seidler RD, Bernard JA, Burutolu TB, Fling BW, Gordon MT, Gwin JT, et al. Motor control and aging: links to age-related brain structural, functional, and biochemical effects. Neurosci Biobehav Rev 2010;34:721-33.

19) Salat DH, Buckner RL, Snyder AZ, Greve DN, Desikan RS, Busa E, et al. Thinning of the cerebral cortex in aging. Cereb Cortex 2004;14: 721-30.

20) Anderson S, Parbery-Clark A, White-Schwoch T, Kraus N. Aging affects neural precision of speech encoding. J Neurosci 2012;32: 14156-64.

21) Zekveld AA, Rudner M, Johnsrude IS, Festen JM, van Beek JH, Rönnberg J. The influence of semantically related and unrelated text cues on the intelligibility of sentences in noise. Ear Hear 2011;32:e16-25.

22) Pichora-Fuller MK. Cognitive aging and auditory information processing. Int J Audiol 2003;42 Suppl 2:2S26-32.

23) Lutman ME, Brown EJ, Coles RR. Self-reported disability and handicap in the population in relation to pure-tone threshold, age, sex and type of hearing loss. Br J Audiol 1987;21:45-58.

24) Matthews LJ, Lee FS, Mills JH, Dubno JR. Extended high-frequency thresholds in older adults. J Speech Lang Hear Res 1997;40:208-14.

25) Wiley TL, Cruickshanks KJ, Nondahl DM, Tweed TS, Klein R, Klein R, et al. Aging and high-frequency hearing sensitivity. J Speech Lang Hear Res 1998;41:1061-72.

26) Gordon-Salant $S$. Hearing loss and aging: new research findings and clinical implications. J Rehabil Res Dev 2005;42(4 Suppl 2):9-24.

27) Snell KB, Frisina DR. Relationships among age-related differences in gap detection and word recognition. J Acoust Soc Am 2000;107: 1615-26.

28) Snell KB. Age-related changes in temporal gap detection. J Acoust Soc Am 1997;101:2214-20.

29) Fitzgibbons PJ, Gordon-Salant S. Age effects on measures of auditory duration discrimination. J Speech Hear Res 1994;37:662-70.

30) He N, Dubno JR, Mills JH. Frequency and intensity discrimination measured in a maximum-likelihood procedure from young and aged normal-hearing subjects. J Acoust Soc Am 1998;103:553-65.

31) Gehr SE, Sommers MS. Age differences in backward masking. J Acoust Soc Am 1999;106:2793-9.

32) Ben-David BM, Tse VY, Schneider BA. Does it take older adults longer than younger adults to perceptually segregate a speech target from a background masker? Hear Res 2012;290:55-63.

33) Letowski T, Poch N. Understanding of time-compressed speech by older adults: effect of discard interval. J Am Acad Audiol 1995;6: 433-9.

34) Tun PA. Fast noisy speech: age differences in processing rapid speech with background noise. Psychol Aging 1998;13:424-34.

35) Killion M, Niquette $P$. What can the pure-tone audiogram tell us about a patient's SNR loss? Hear J 2000;53:46-53.

36) Goŕdon-Salant S, Fitzgibbons PJ. Temporal factors and speech recognition performance in young and elderly listeners. J Speech Hear Res 1993;36:1276-85.

37) Dubno JR, Dirks DD, Morgan DE. Effects of age and mild hearing loss on speech recognition in noise. J Acoust Soc Am 1984;76:87-96.

38) Hällgren M, Larsby B, Lyxell B, Arlinger S. Speech understanding in quiet and noise, with and without hearing aids. Int J Audiol 2005;44: 574-83.

39) Kochkin, S. MarkeTrak V. Baby Boomers spur growth in potential market, but penetration rate declines. Hear J 1999;52:33-48.

40) Labadie RF, Carrasco VN, Gilmer CH, Pillsbury HC 3rd. Cochlear implant performance in senior citizens. Otolaryngol Head Neck Surg 2000;123:419-24.

41) Chatelin V, Kim EJ, Driscoll C, Larky J, Polite C, Price L, et al. Cochlear implant outcomes in the elderly. Otol Neurotol 2004;25:298-301. 
42) Ouayoun M, Mérite-Drancy A, Monneron L, Fugain C, Chouard CH, Meyer B. [Cochlear implant in the aged patient]. Ann Otolaryngol Chir Cervicofac 1998;115:135-9.

43) Boothroyd A. Hearing aid accessories for adults: the remote FM microphone. Ear Hear 2004;25:22-33.

44) Anderson S, White-Schwoch T, Parbery-Clark A, Kraus N. Reversal of age-related neural timing delays with training. Proc Natl Acad Sci
U S A 2013;110:4357-62.

45) Smith GE, Housen P, Yaffe K, Ruff R, Kennison RF, Mahncke HW, et al. A cognitive training program based on principles of brain plasticity: results from the Improvement in Memory with Plasticitybased Adaptive Cognitive Training (IMPACT) study. J Am Geriatr Soc 2009;57:594-603. 